\title{
La información y el conocimiento y su relación con la calidad de los servicios bibliotecarios Un estudio de caso
}

\author{
Fredy Eduardo Vásquez Rizo \\ Universidad Autónoma de Occidente, Facultad de Comunicación y Ciencias Sociales, Departamento de Ciencias de la \\ Comunicación, Colombia | fvasquez@uao.edu.co / http://orcid.org/oooo-ooo3-1398-6174
}

\author{
Jesús Gabalán Coello \\ Universidad Católica de Pereira, Vicerrectoría Académica, Colombia | jesus.gabalan@ucp.edu.co / \\ http://orcid.org/oooo-0001-7674-8849
}

\begin{abstract}
Resumen
Este artículo presenta un análisis realizado en la biblioteca de la Universidad Autónoma de Occidente, ubicada en Santiago de Cali, Colombia, acerca de la calidad de los servicios prestados y su relación con la información presente en el acervo bibliográfico y su posibilidad de generar conocimiento en los usuarios. Para ello, se llevó a cabo un estudio de corte cuantitativo, el cual permitió revisar, con base en unas preguntas cerradas, el comportamiento de algunas variables asociadas a dichos servicios a partir de las expectativas de los usuarios, señalando para la biblioteca algunos aspectos de mejora, reconociendo su importancia como dependencia crucial para el desarrollo de la institución y para contestar los requerimientos y necesidades de información en el marco de la sociedad de la información y el conocimiento.
\end{abstract}

Information and knowledge and their relation to the quality of library services. A case study

\begin{abstract}
This article presents an analysis carried out in the library of Universidad Autónoma de Occidente, located in Santiago de Cali, Colombia, about the quality of the services provided and their relation with the current information in the bibliographic collection and its possibility of generating knowledge in the users. For this, a quantitative study was carried out, which allowed to review, based on some closed questions, the behavior of some variables associated with these services based on the expectations of the users, pointing out for the library some aspects of improvement, recognizing its importance as a crucial place for the development of the institution and for answering the requirements and information needs within the framework of the information and knowledge society.
\end{abstract}

Palabras clave

Servicios bibliotecarios Información Conocimiento Biblioteca universitaria Percepciones

\section{Keywords}

Library services Information Knowledge University library Perceptions 


\section{Introducción}

Los servicios brindados por las bibliotecas se han venido transformando a lo largo del tiempo. Esto a raíz de la aparición de las Tecnologías de la Información y la Comunicación - TIC, entre otros aspectos, situación que ha influido en sus procesos asociados al tratamiento de la información (Li y Liu, 2019), con un alto grado de afectación en la eficacia y calidad asociadas a la prestación de estos mismos servicios.

Es que es tanta la relevancia de la información en el mundo de hoy (Lynne y Rowe, 2018), y más en estos escenarios (Thanuskodi, 2019), que las bibliotecas tienen, prontamente, que idear maneras de apropiar y distribuir información, a partir de eficientes estrategias de gestión que les permitan fortalecer sus recursos, al tiempo que se preocupan por la satisfacción de las necesidades informacionales de sus usuarios.

Por lo tanto, las bibliotecas son concebidas en el presente como recintos que han pasado de ser simples depósitos de información a convertirse en dependencias neurálgicas (Gavilán, 2008), encargadas de producir conocimiento. Todo un proceso de transformación que necesariamente involucra la percepción de los usuarios, que son finalmente quienes deben reconocer el desempeño de la labor cumplida, en términos de los servicios ofrecidos por la biblioteca, y si estos contribuyen a generar valor agregado en sus acervos personales de conocimiento.

Es por todo esto que el presente artículo pretende analizar cuál es el grado de satisfacción en la calidad de los servicios prestados por una biblioteca universitaria moderna (referencia, préstamo de reserva, préstamo externo, renovación de materiales, multas y apartados, uso de espacios físicos, entre otros), con la intención de identificar si verdaderamente los usuarios perciben dicha transformación, desde una información desbordante hacia un conocimiento útil, pertinaz y concreto.

Biblioteca que forma parte del sistema académico de la Universidad Autónoma de Occidente, institución que cuenta con más de 8000 estudiantes y alrededor de 17000 egresados, todos ellos con posibilidad de ser usuarios de las instalaciones del centro de información, así como sus 228 profesores de planta, distribuidos en cinco (5) facultades: Comunicación Social, Ingenierías, Ciencias Económicas y Administrativas, Ciencias Básicas y Humanidades e Idiomas.

\section{Revisión de la literatura}

\subsection{Las bibliotecas y sus servicios}

Unas de las organizaciones sociales y culturales con mayor historia que existen actualmente son las bibliotecas. Entidades con gran reconocimiento por parte de la sociedad, como promotoras del desarrollo intelectual a través de la recuperación y acceso democrático a la información, a partir de diversos medios y recursos (Michener, Contreras y Niskier, 2018). Elementos instrumentales que inciden en la calidad del servicio que se brinda, en la satisfacción del usuario, en la pronta respuesta a un requerimiento, en la asimilación del entorno exterior, en la innovación y en el aprendizaje, entre muchos otros aspectos.

Es por esto que el valor agregado de estas entidades estriba en la manera como ellas logran asumir estas responsabilidades a partir del cumplimiento adecuado de sus servicios (Melnik, Pereira y Somma, 2005). Servicios que confluyen en intentar satisfacer las necesidades de información de los usuarios, las cuales son cada vez más exigentes, debido al ritmo vertiginoso del tiempo y al exceso informativo característico 
de la sociedad de la información y el conocimiento, características que hacen que los servicios de información deban ser cada vez más responsables con sus materiales y contenidos (García-Sánchez, 2005).

\subsection{La información y el conocimiento en los servicios de las bibliotecas}

Dichos materiales y contenidos tienen como epicentro a la información, que es una sumatoria de datos con significancia y sentido (Laudon y Laudon, 2014), con cierto grado de utilidad para las acciones humanas (Calvo-Manzano-Villalon et al., 2007; Milán-Pérez, Guilarte y Domínguez-Dreke, 2011), que se potencia a través de efectivos procesos de gestión (Vásquez-Rizo, Rodríguez-Muñoz y Gómez-Hernández, 2019), tendientes a la generación de conocimiento.

Proceso que implica la transformación del elemento informacional hacia el elemento cognitivo (Poikela, 2004; Alaarj, Abidin-Mohamed y Bustamam, 2016) en el escenario específico donde se lleve a cabo dicha gestión. Tal es el caso de las bibliotecas, donde su acervo informativo, al ser gestionado correctamente, les permite a los usuarios adquirir un conocimiento sobre un respectivo tema, dando solución a sus necesidades de información, fortaleciendo su aprendizaje (Gil-López y Carrillo-Gamboa, 2016; Pozo-Municio, 2018).

Y es esta habilidad de crear, asimilar y difundir conocimiento la que es reconocida hoy en día como una capacidad estratégica clave para el óptimo desarrollo y evaluación de los servicios ofrecidos y para la percepción de generación de valor agregado y ventaja competitiva (Moraga-Vega, 2012; Vásquez-Rizo y Gabalán-Coello, 2017; Khurshid, Zheng y Muhammad, 2019) por parte de los usuarios de la información.

\subsection{Las bibliotecas y la sociedad de la información y el conocimiento}

En el contexto de la actual sociedad de la información y el conocimiento, se asiste a una nueva misión y dimensión de la biblioteca como organización sistémica, compleja, flexible y con capacidad de adaptarse a los nuevos modelos de enseñanza-aprendizaje, más versátiles, tecnológicos y personalizados, centrados en la transferencia de habilidades y competencias informativas para la toma de decisiones y para el uso, aprovechamiento y difusión de la información, como sustento para la adquisición de conocimientos (Suaiden, 2018).

Bajo esta realidad, se puede afirmar que nunca como ahora, ha existido un movimiento tan fuerte en favor de los servicios bibliotecarios (Wójcik, 2019), del fortalecimiento de la estructura online y offline de las bibliotecas (Fernández-Ramos, 2016) y de la cualificación de su personal (Cervera-Farré et al., 2015). Aspectos estos que necesariamente repercuten en la calidad de dichos servicios (y de la dependencia), siendo los usuarios quienes determinan finalmente la aceptación o no de dicha particularidad, reconociendo y evaluando aspectos como la biblioteca misma (lugar), el servicio (proceso), los implicados en el servicio (personal), los insumos para el servicio (recursos) y los efectos del servicio (resultados) (Pérez-Pupo et al., 2014).

Es así como se puede decir que el objetivo de toda biblioteca moderna debe consistir entonces en ofrecer servicios bibliotecarios de calidad, en un ambiente que estimule y facilite al usuario la adquisición de información pertinente, la lectura, la investigación, la construcción de conocimiento y el aprendizaje. Pues finalmente será este usuario quien evalúe el accionar de la biblioteca, de acuerdo con su nivel de satisfacción en relación con el servicio prestado (Escalona-Ríos, 2004), y por ello es fundamental que sea este mismo usuario quien reconozca a la biblioteca como un puente transformador de su información latente en un nuevo conocimiento. 


\section{Métodos y materiales}

Este estudio fue de carácter cuantitativo y para su desarrollo fue necesario crear un instrumento de recolección de datos (encuesta) que se aplicó a una muestra específica (probabilística aleatoria) de usuarios de los servicios de la biblioteca objeto de estudio. Según Busha (1990), en bibliotecología, los métodos de investigación por encuesta son las técnicas más adecuadas. Posición compartida, de manera general, por Hernández-Sampieri, Fernández-Collado y Baptista-Lucio (2014), quienes consideran a la encuesta como una técnica acertada para ejercicios de este tipo.

Para ello se realizó, previo a su aplicación, un testeo de confiabilidad, validez y dominio de contenido, a través de pruebas estadísticas descriptivas, lo que permitió concretar las variables a medir. Esto posibilitó definir una serie de preguntas cerradas, cuya colinealidad mostró relación entre ellas pero no exclusión o sobreposición. Y el procedimiento de codificación que se utilizó fue la tabulación de datos.

Así, se establecieron variables relacionadas principalmente con el tipo de usuario, el género, la frecuencia de uso de la biblioteca (presencial o virtual), las razones de uso de la biblioteca, las características del espacio, la infraestructura, la locación, las condiciones ambientales, la disponibilidad del material bibliográfico, la actualización del material bibliográfico, la mediación tecnológica, la atención del personal, la difusión de información, los servicios ofrecidos y la relación entre todas las variables mencionadas con dichos servicios.

Adicional a esto, la investigación fue de tipo no experimental, definida por HernándezSampieri, Fernández-Collado y Baptista-Lucio (2014) como aquella que se realiza sin manipular deliberadamente las variables, observando el fenómeno tal y como se da en su contexto natural, para después analizarlo. Esto sugirió un diseño transeccional descriptivo cuyo procedimiento consistió en medir o ubicar a un grupo de fenómenos en una variable o concepto y proporcionar su descripción. Lo que permitió definir dos tipos de variables comparativas: las expectativas de los usuarios y la calidad de los servicios.

Posteriormente, se aplicó la encuesta aleatoriamente a la población sujeto de estudio, un total de 1.485 personas (usuarios de la biblioteca), con la intención de determinar su satisfacción en relación con los servicios. Muestra que surgió de revisar las planillas de usuarios del último año y estadísticamente definir dicho número como representativo. Aquí vale la pena mencionar que este estudio se llevó a cabo en la biblioteca de una universidad privada, ubicada en Santiago de Cali, Colombia, abierta a todo público, condición que posibilita el libre acceso de sus servicios a toda la comunidad y no solo a los miembros de la Institución de la que forma parte (como constará más adelante).

\section{Resultados}

Con base en la cantidad de usuarios encuestados, el Gráfico 1 presenta el porcentaje de ocupación o relación de estos con la biblioteca universitaria. Aquí se evidencia que la mayoría de ellos son profesionales (distintos a la docencia), mientras que los restantes son profesores y estudiantes de preparatoria o posgrado, quienes constituyen el $55 \%$ de los usuarios, aspecto que remarca el carácter académico de la biblioteca.

Sin embargo, llama la atención el porcentaje de usuarios clasificados como "profesionales", puesto que esto puede tener dos lecturas, ambas con posibilidad de profundización: 1. Que la biblioteca es visitada continuamente por personal externo al campus y 


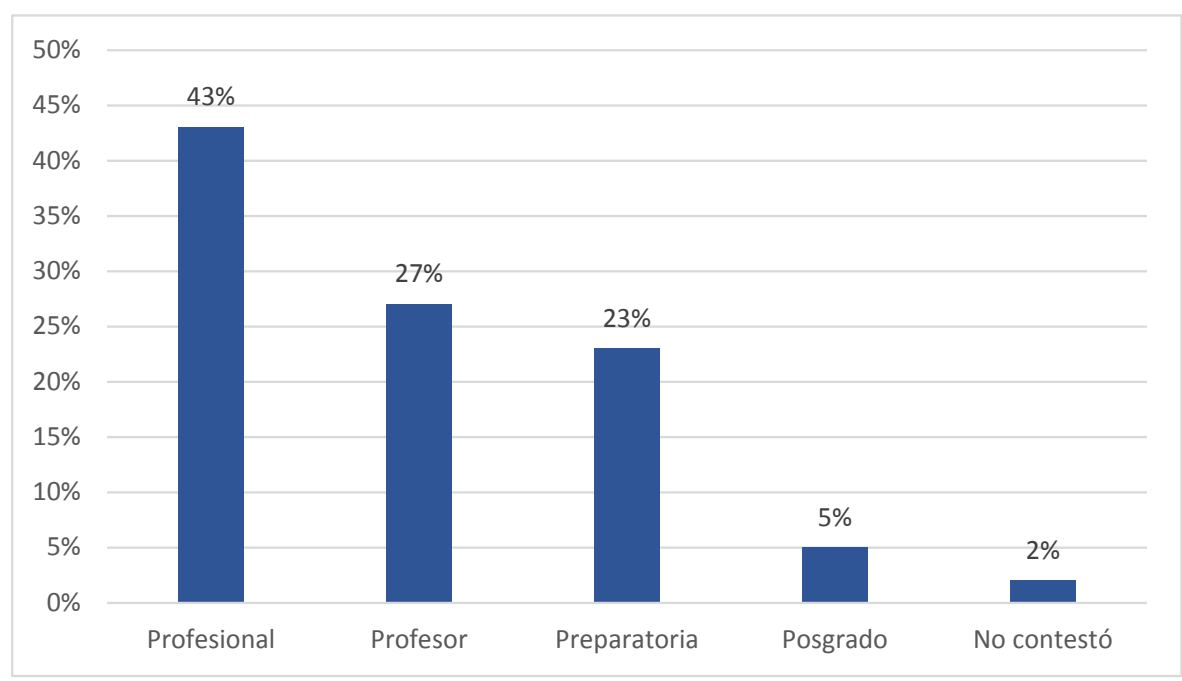

Fuente: elaboración propia

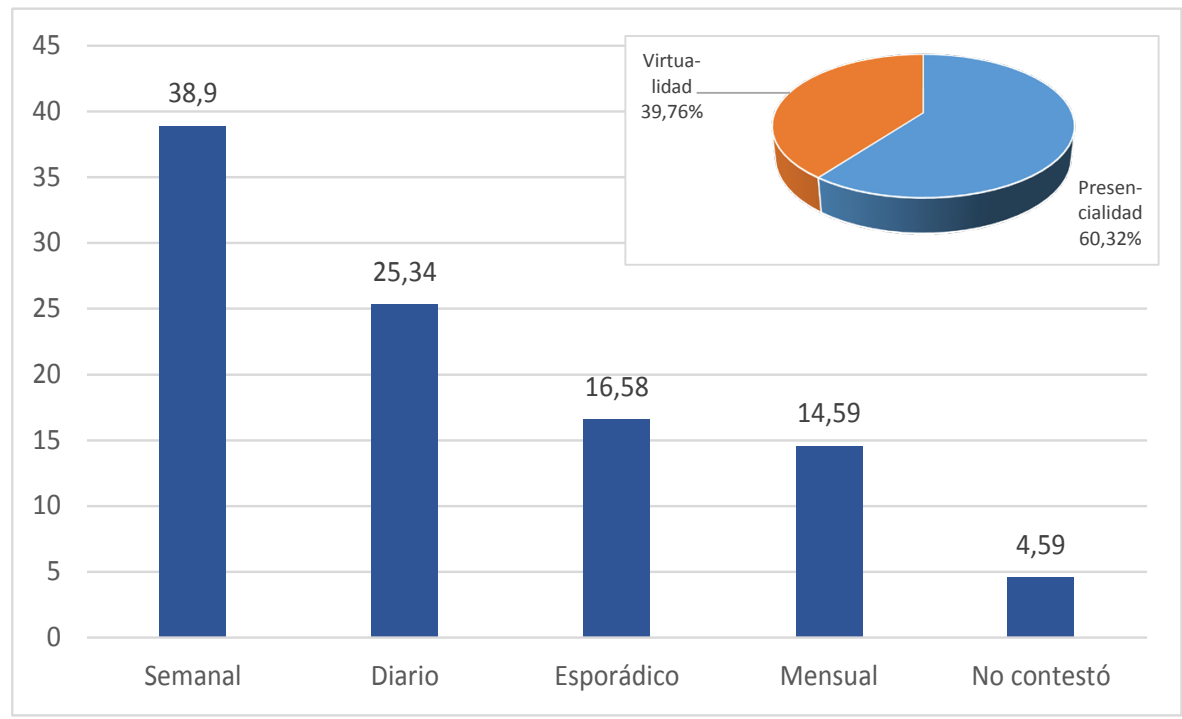

Fuente: elaboración propia

2. Que la biblioteca necesita promover un mayor número de actividades que acerquen mucho más dicha dependencia y sus servicios a la presente comunidad universitaria.

A continuación, se muestran los gráficos asociados a las variables más representativas, las cuales permiten resolver el objetivo del estudio.

En términos de asistencia a las instalaciones de la biblioteca (físicas o virtuales), se pudo constatar que más del $60 \%$ de los usuarios la visitan semanal o diariamente. Cifra que es representativa acerca de la aceptación que dicha dependencia tiene para la resolución de sus necesidades de información (Gráfico 2).
Gráfico 1. Ocupación o relación de los usuarios con la biblioteca
Gráfico 2. Frecuencia de uso de la biblioteca 


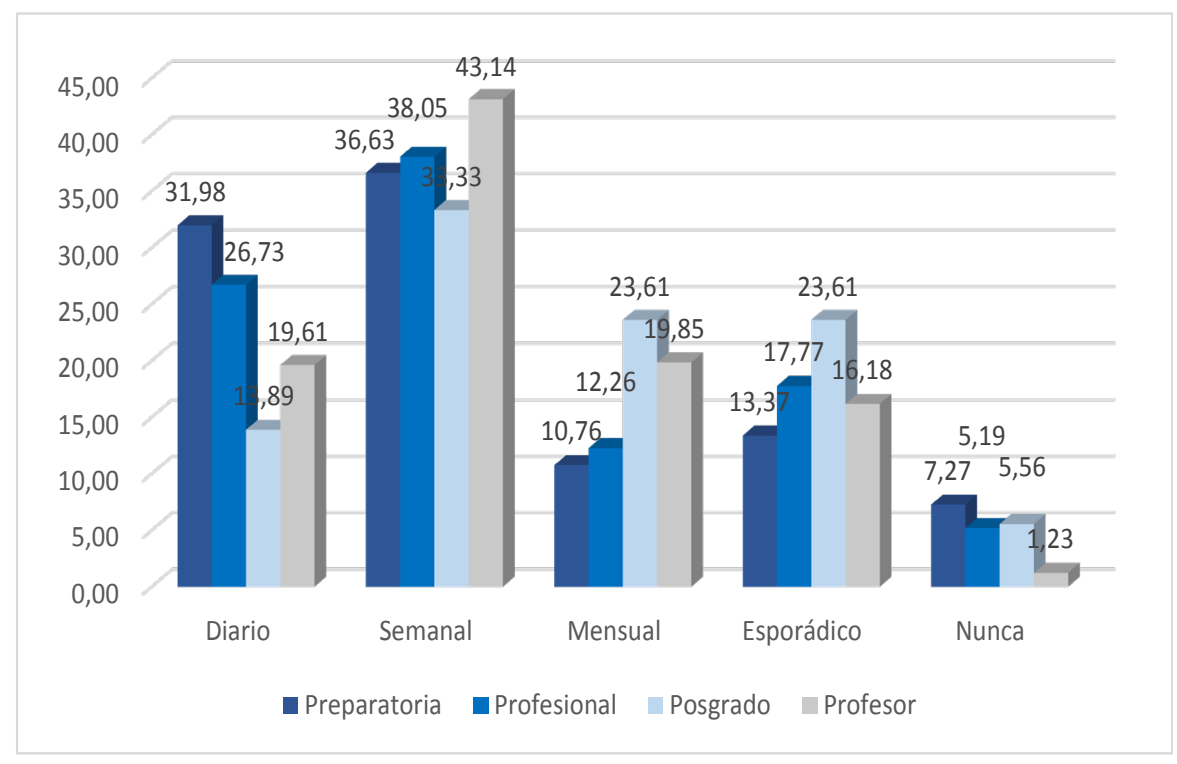

Fuente: elaboración propia

Adicional a esto, en el mismo Gráfico se nota una tendencia hacia la virtualidad, lo que implica que la biblioteca cuenta con alternativas digitales aceptables para el ofrecimiento de sus servicios, pero también que debe atender lo que está ocurriendo con sus tácticas asociadas a la presencialidad. Aunque dicha situación se puede deber al rumbo informacional hacia lo virtual de la misma sociedad (Damian, 2017).

Ahora bien, se sabe que es distinto el comportamiento de los cuatro grupos de usuarios. Por lo tanto, en el Gráfico 3 se expone la frecuencia de visita de cada uno. En ella se puede apreciar que el más alto pico se encuentra en los profesores que la visitan semanalmente (43,14\%), seguido por los profesionales en este mismo periodo de tiempo (38,05\%).

Llama la atención que los estudiantes de posgrado son quienes menos uso permanente hacen de los servicios de la biblioteca, condición que va un poco en contravía con lo que se espera de sus necesidades de información, pues es reconocido que en este nivel formativo los requerimientos de información y la necesidad de construcción de nuevo conocimiento son constantes (Al Qallaf, 2019).

Con respecto a las condiciones físicas de la biblioteca, el Gráfico 4 establece que la iluminación, el aseo y la ubicación son los ítems mejor calificados (excelente), mientras que el silencio es el que menos nivel de aceptación evidencia. Este es un punto importante a tener en cuenta en la prestación adecuada de los servicios, pues el silencio es un elemento determinante para el buen funcionamiento de una biblioteca (Mourer y Karadjova, 2017).

Debido a que se trata de una biblioteca universitaria y que muchos de sus servicios se enfocan en que esta sea un espacio adecuado para el estudio, tanto presencial como colaborativamente en red, el Gráfico 5 enfatiza en la percepción de los usuarios al respecto, dejando ver que casi el 80\% de ellos califica como óptima o buena esta condición. Sin embargo, vale la pena que la biblioteca revise cómo esta se podría 


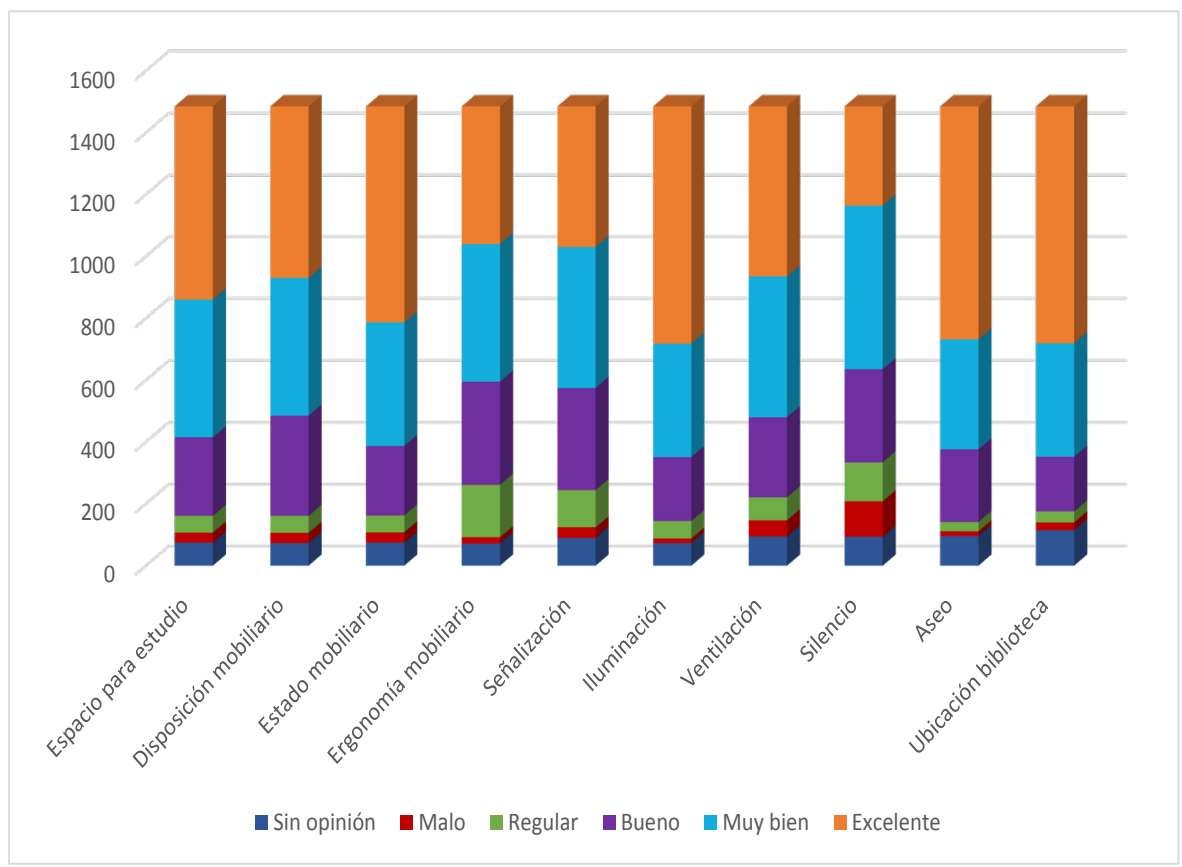

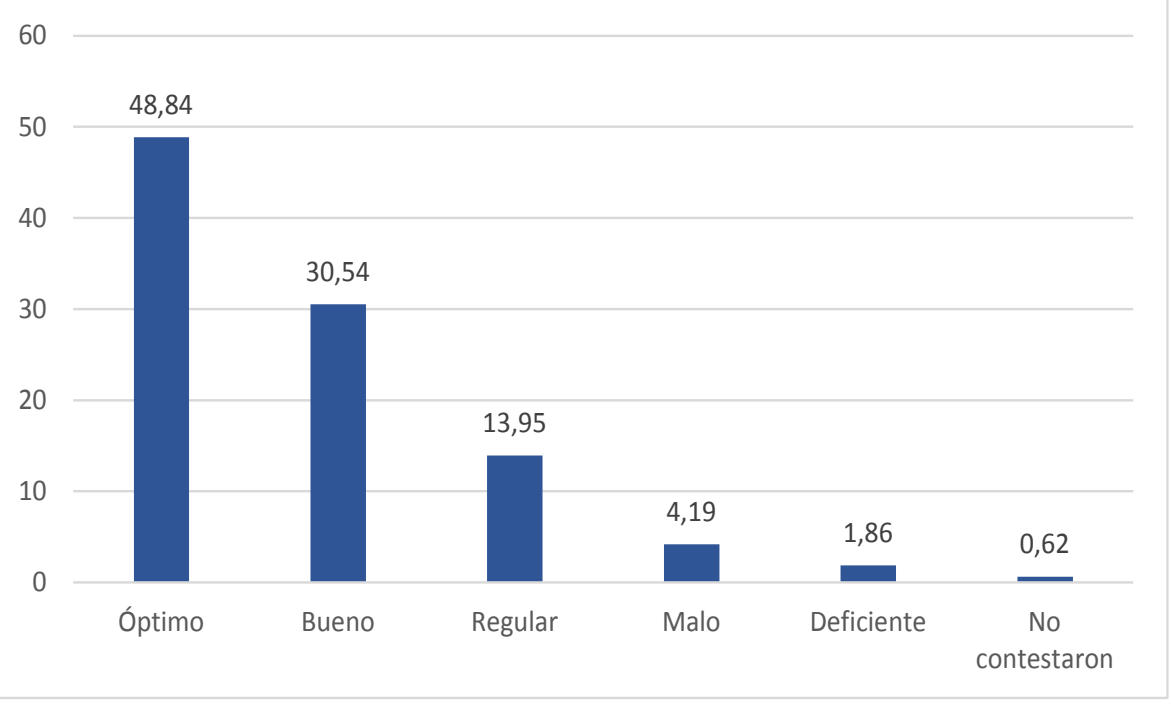

Fuente: elaboración propia
Gráfico 5. Calificación de la condición "espacio de estudio"

mejorar para aquellas personas que aún no la consideran adecuada (el 20\%, sumando las opciones: regular, malo y deficiente).

A continuación, el Gráfico 6 condensa los principales servicios ofrecidos por la biblioteca. En ella se observa que la percepción de los usuarios es relativamente positiva, pues todos los ítems se encuentran por encima de un 70\% de aceptación, siendo "préstamo externo" el servicio de mejor calificación y "multas y apartados" el de menor. 


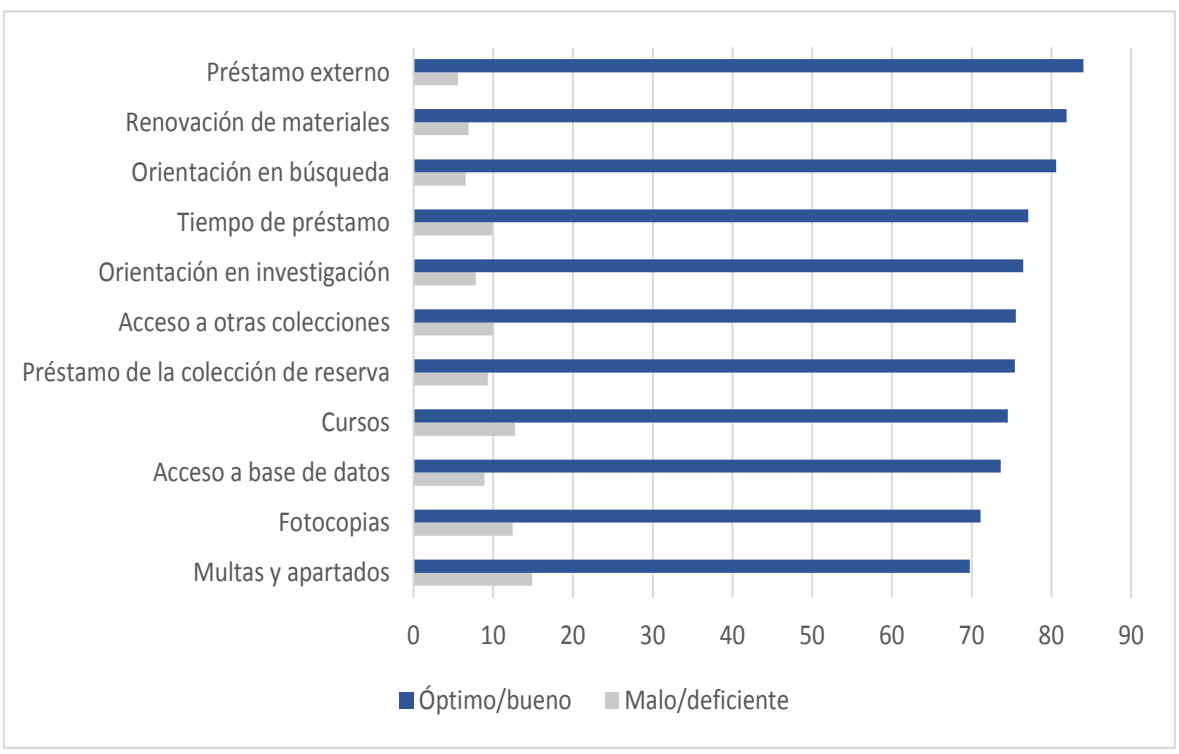

Fuente: elaboración propia

Aquí, adicional a "multas y apartados", que tal vez tenga algún sentido lógico en su animadversión, se debe revisar por parte de la biblioteca universitaria el servicio asociado a los cursos de capacitación impartidos, pues existe un pequeño porcentaje de usuarios que no está de acuerdo con la manera como estos se están llevando a cabo, siendo este un servicio crucial para la alfabetización acerca de los demás servicios prestados por las bibliotecas (Gómez-Hernández y Licea-De-Arenas, 2005).

Para terminar, es importante mostrar la percepción de los usuarios en cuanto a las características asociadas a los recursos bibliográficos (servicio transversal a los anteriormente presentados y fundamental para cualquier biblioteca en materia de su calidad), a partir de la cual se puede analizar que si bien se manifiesta un desempeño aceptable, es necesaria la realización de algunos correctivos, pues una biblioteca óptima debería tener todos sus ítems por encima del máximo valor posible (Universidad de Los Andes, 2016), que en este caso sería "excelente" (Gráfico 7).

\section{Discusión}

Este estudio permitió ampliar los conocimientos en relación con el concepto de los servicios y las implicaciones que estos conllevan para las actuales bibliotecas, siendo un elemento importante en el ofrecimiento de los intangibles asociados a los bienes tradicionales que son los que normalmente el usuario percibe.

Es decir que los servicios se constituyen en aquellos aspectos que le permiten a la biblioteca ampliar su panorama de desempeño, potenciar sus capacidades y demostrar sus habilidades adaptativas e innovadoras, para hacer frente a las continuas y constantes transformaciones del mundo globalizado, evidentes en las variaciones de los contextos y en las disímiles necesidades de los usuarios.

Es por esto que las bibliotecas modernas, para desarrollar y optimizar sus diferentes servicios, deben necesariamente conformar estructuras sinérgicas, coherentes y articuladas que integren sus principales procesos en función del progreso. Progreso dado por la satisfacción manifiesta del usuario, quien ya no es esa persona estática sino un sujeto activo y partícipe en la auscultación de su propia información y en 


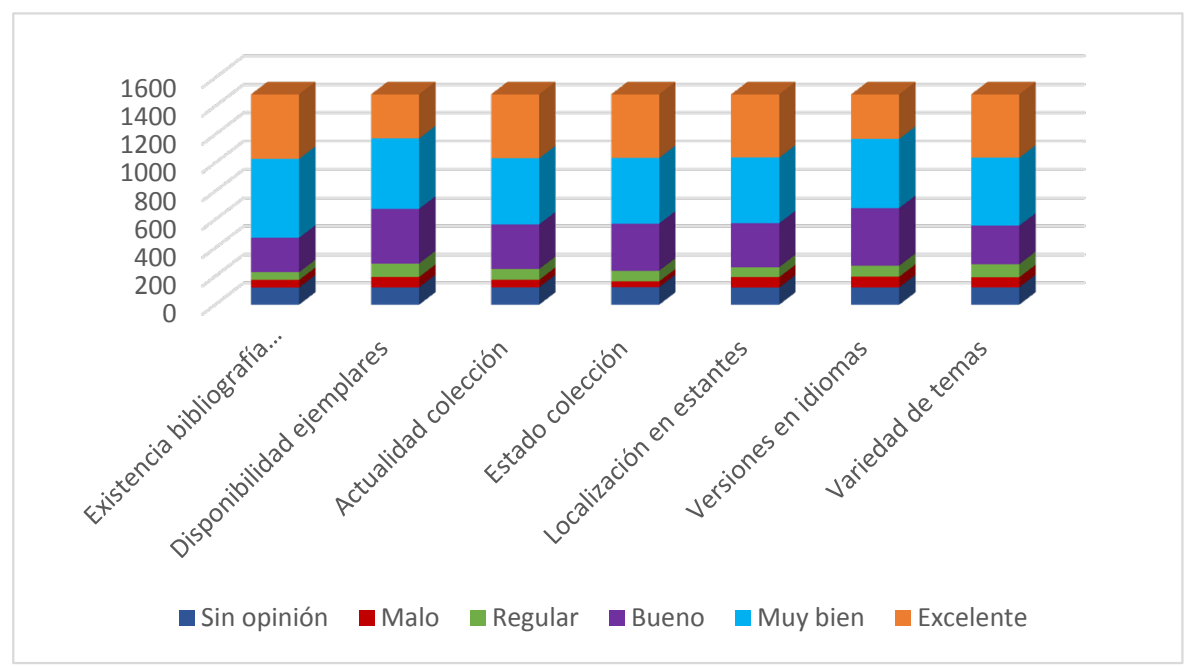

Fuente: elaboración propia
Gráfico 7. Percepción de los recursos bibliográficos

la construcción de su propio conocimiento. Pues es finalmente ese usuario quien orienta a la biblioteca en términos de hacia dónde debe dirigir su oferta de servicios.

\section{Conclusiones}

En la actual era de la información y el conocimiento las bibliotecas, como centros receptores y difusores de información y promotores de conocimiento, se han convertido en espacios fundamentales y estratégicos. En este estudio se pudo constatar cómo una biblioteca universitaria realiza un análisis enfocado en fortalecer y potenciar sus servicios (apuntando hacia la calidad), con base en la percepción que de estos tienen sus usuarios, con la intención de solventar adecuadamente sus numerosas y heterogéneas necesidades de información.

Es así como se detectaron como principales servicios asociados: el uso y las condiciones de los espacios físicos, el préstamo externo, la renovación de materiales, la orientación en las búsquedas, los préstamos de la colección de reserva, el acceso a las bases de datos, las multas y apartados, los recursos bibliográficos, entre otros.

Y para revisar la percepción de los usuarios respecto a ellos, se diseñó y aplicó un instrumento de tipo cuantitativo, conformado por un cuestionario de preguntas cerradas, el cual hizo posible conocer las distintas percepciones de los usuarios, en torno a su biblioteca, lo que permitió contrastar las expectativas de los usuarios (estado ideal) con la calidad de los servicios (estado actual). Situación que posibilitó identificar elementos de oportunidad, mejora y desarrollo bibliotecológico.

Como se puede apreciar, si bien se trató de un estudio muy particular, el mismo permitió identificar los servicios ofrecidos y reconocer el pensamiento de los usuarios, apuntando a establecer qué tan cercanos o lejanos se encuentran dichos servicios en relación con el proceso de transformación que debe sufrir la información presente en la biblioteca hacia la obtención de un conocimiento concreto por parte de los usuarios, tendiente a satisfacer sus requerimientos y necesidades. Todo ello enmarcado en la fundamental función que debe cumplir hoy en día una biblioteca que quiere ser partícipe de la sociedad de la información y el conocimiento. 


\section{Q Referencias Bibliográficas}

"Al Qallaf, Charlene. 2019. Information literacy assessment of incoming students in an information studies graduate program. En Global Knowledge, Memory and Communication. Vol. 68, no. 3, 223-241. <https://doi.org/10.1108/ GKMC-07-2018-0062>

"Alaarj, Saad; Zainal Abidin-Mohamed y Ummi Salwa Brinti Ahmad Bustamam. 2016. Mediating role of trust on the effects of knowledge management capabilities on organizational performance. En Procedia - Social and Behavioral Sciences. Vol. 235, 729-738. <https://doi.org/10.1016/j.sbspro.2016.11.074>

» Busha, Charles. 1990. Métodos de investigación en bibliotecología: técnicas e interpretación. México D.F.: Universidad Nacional Autónoma de México (UNAM).

»Calvo-Manzano-Villalon, José; Joaquín Cervera-Bravo; Luis Fernández-Sanz y Ma Piattini-Velthuis. 2007. Análisis y diseño detallado de aplicaciones informáticas de gestión. México D.F.: Alfaomega Grupo Editor - Rama.

»Cervera-Farré, Albert; Elisabet Cervera-Biedma; Cristina López-Pérez; Gema Santos-Hermosa y Cristina Vaquer-Suñer. 2015. Hacia el Liaison Librarian: transformación de servicios bibliotecarios para dar apoyo a la docencia en la Universitat Oberta de Catalunya (UOC). En El Profesional de la Información. Vol. 24, no. 2, 121-129. <https://doi.org/10.3145/epi.2015.mar.05>

"Damian, leda Pelógia Martins. 2017. Análise do serviço de referência virtual em bibliotecas universitárias. En Transinformação. Vol. 29, no. 3, 221-232. <https:// doi.org/10.1590/2318-08892017000300001>

"Escalona-Ríos Lina. 2004. La evaluación del servicio de consulta ¿calidad o cantidad? En Biblioteca Universitaria. Vol. 7, no. 1, 12-22. <http://eprints.rclis. org/7638/1/Vol7No1_ene.jun2004_p_12-22.pdf> [Consulta: 20 junio 2019].

»Fernández-Ramos, Andrés. 2016. Los servicios bibliotecarios en la web: retos y oportunidades. En Investigación Bibliotecológica. Vol.30, no. 69, 11-15. <https:// doi.org/10.1016/j.ibbai.2016.04.010>

"García-Sánchez, Jaquelinne. 2005. Conocimiento de los servicios y recursos bibliográficos-electrónicos por parte de los alumnos, ofrecidos por la biblioteca del ITESM Campus León. Monterrey: Instituto Tecnológico y de Estudios Superiores de Monterrey (ITESM). 93 p. Tesis de maestría. <https://repositorio.tec.mx/ handle/11285/567310> [Consulta: 20 junio 2019].

» Gavilán, César Martín. 2008. Bibliotecas universitarias: concepto y función. Los CRAl. <http://eprints.rclis.org/14816/1/crai.pdf> [Consulta: 2 julio 2019].

»Gil-López, Alfonso y Francisco Javier Carrillo-Gamboa. 2016. Knowledge transfer and the learning process in Spanish wineries. En Knowledge Management Research \& Practice. Vol. 14, no. 1, 6o-68. <https://doi.org/10.1057/kmrp.2014.12>

»Gómez-Hernández, José Antonio y Judith Licea-De-Arenas. 2005. El compromiso de las bibliotecas con el aprendizaje permanente. La alfabetización informacional. En Perelló, Javier Gimeno y Pedro López-López, ed. Información, conocimiento y bibliotecas en el marco de la globalización neoliberal. Gijón: Ediciones Trea. p. 145-18o.

" Hernández-Sampieri, Roberto; Carlos Fernández-Collado y Pilar Baptista-Lucio. 2014. Metodología de la investigación (6a. ed.). México D.F.: McGraw-Hill. 
» Khurshid, Ahmad; JianMing Zheng y Rafi Muhammad. 2019. Assessing the literature of Knowledge Management (KM) in the field of library and information science. En Information Discovery and Delivery. Vol. 47, no. 1, 35-41. <https://doi. org/10.1108/IDD-06-2018-0021>

» Laudon, Kenneth y Jane Laudon. 2014. Management information systems. Managing the digital firm (13th ed.). Harlow: Pearson Education Limited.

» Li, Yuelin y Chang Liu. 2019. Information resource, interface, and tasks as user interaction components for digital library evaluation. En Information Processing \& Management. Vol. 56, no. 3, 704-720. <https://doi.org/10.1016/j. ipm.2018.10.012>

"Lynne, Markus y Frantz Rowe. 2018. Is it changing the world? Conceptions of causality for information systems theorizing. En MIS Quarterly. Vol. 42, no. 4, 1255-128o. <https://doi.org/10.25300/MISQ/2018/12903>

»Melnik, Diana; María Elina Pereira y Pablo Somma. 2005. Bases para la administración de biblioteca: organización y servicios. Buenos Aires: Alfagrama Ediciones.

" Michener, Gregory; Evelyn Contreras e Irene Niskier. 2018. From opacity to transparency? Evaluating access to information in Brazil five years later. En Revista de Administração Pública. Vol. 52, no. 4, 610-629. <https://doi. org/10.1590/0034-761220170289>

" Milán-Pérez, Andrés; Aniripsa Felipe Guilarte y María Teresa Domínguez-Dreke. 2011. Registro de datos primarios, herramienta de gestión de la información. En Ciencias de la Información. Vol. 42, no. 1,39-44. <http://www.redalyc.org/articulo.oa?id=181418901006 > [Consulta: 3 julio 2019].

»Moraga-Vega, Katia. 2012. Competitividad e indicadores en la biblioteca de la Universidad para la Paz. En Revista e-Ciencias de la Información. Vol. 2, no. 1, 111. <https://doi.org/10.15517/ECl.V2l1.1218>

"Mourer, Marissa y Katia Karadjova. 2017. Dare to share the silence: Tools and practices of contemplative pedagogy in a library brain booth. Cham: Springer International Publishing.

»Pérez-Pupo, Annarelis; Aracely Lores-Cruz; Lisbet Pérez-Randiche; Arelys Lores-Cruz y Yuna Díaz-Rojas. 2014. Evaluación de la calidad de los servicios bibliotecarios: un reto actual en bibliotecas médicas. En Correo Científico Médico. Vol. 18, no. 3, 577-592. <http://scielo.sld.cu/pdf/ccm/v18n3/ccm26314.pdf> [Consulta: 2 julio 2019].

»Poikela, Esa. 2004. Developing criteria for knowing and learning at work: towards context-based assessment. En Journal of Workplace Learning. Vol. 16, no. 5, 267-274. <https://doi.org/10.1108/13665620410545543>

»Pozo-Municio, Juan Ignacio. 2018. Psicología del aprendizaje humano: adquisición de conocimiento y cambio personal. Madrid: Ediciones Morata.

"Suaiden, Emir José. 2018. La biblioteca pública y las competencias del siglo XXI. En El Profesional de la Información. Vol. 27, no. 5, 1136-1144. <https://doi. org/10.3145/epi.2018.sep.17>

" Thanuskodi, Shanmugam. 2019. Information literacy skills among library and information science professionals in India. En Library Philosophy \& Practice. Vol. 2126, 1-24. <https://digitalcommons.unl.edu/cgi/viewcontent.cgi?article=5499 \&context=libphilprac> [Consulta: 30 junio 2019]. 
"Universidad de Los Andes. 2016. Análisis de recursos bibliográficos. <https:// planeacion.uniandes.edu.co/dmdocuments/FT-\%20Analisis\%20de\%2orecursos\%2obibliograficos\%20(1).pdf> [Consulta: 30 junio 2019].

»Vásquez-Rizo, Fredy Eduardo y Jesús Gabalán-Coello. 2017. Agregando valor a las IES a través de la búsqueda y selección de información. En Prisma Social. Vol. 18, no. 1, 592-602. <https://dialnet.unirioja.es/servlet/articulo?codigo=6220245> [Consulta: 3 julio 2019].

»Vásquez-Rizo, Fredy Eduardo; José Vicente Rodríguez-Muñoz y José Antonio Gómez-Hernández. 2019. La gestión de información para medir la capacidad investigadora en una institución de educación superior. En Revista Espacios. Vol. 40, no. 8, 18-30. <https://revistaespacios.com/a19v4ono8/a19v4ono8p18. pdf> [Consulta: 8 julio 2019].

»Wójcik, Magdalena. 2019. How to design innovative information services at the library? En Library Hi Tech. Vol. 37, no. 2, 138-154. <https://doi.org/10.1108/ LHT-07-2018-0094> 